\title{
Direct numerical simulations of turbulent flow over a permeable wall using a direct and a continuum approach
}

\author{
W. P. Breugem and B. J. Boersma \\ J.M. Burgers Center for Fluid Dynamics, Delft University of Technology, Leeghwaterstraat 21, \\ 2628 CA Delft, The Netherlands
}

(Received 1 September 2004; accepted 26 October 2004; published online 6 January 2005)

\begin{abstract}
A direct numerical simulation (DNS) has been performed of turbulent channel flow over a three-dimensional Cartesian grid of $30 \times 20 \times 9$ cubes in, respectively, the streamwise, spanwise, and wall-normal direction. The grid of cubes mimics a permeable wall with a porosity of 0.875 . The flow field is resolved with $600 \times 400 \times 400$ mesh points. To enforce the no-slip and no-penetration conditions on the cubes, an immersed boundary method is used. The results of the DNS are compared with a second DNS in which a continuum approach is used to model the flow through the grid of cubes. The continuum approach is based on the volume-averaged Navier-Stokes (VANS) equations [S. Whitaker, "The Forchheimer equation: a theoretical development," Transp. Porous Media 25, 27 (1996)] for the volume-averaged flow field. This method has the advantage that it requires less computational power than the direct simulation of the flow through the grid of cubes. More in general, for complex porous media one is usually forced to use the VANS equations, because a direct simulation would not be possible with present-day computer facilities. A disadvantage of the continuum approach is that in order to solve the VANS equations, closures are needed for the drag force and the subfilter-scale stress. For porous media, the latter can often be neglected. In the present work, a relation for the drag force is adopted based on the Irmay ["Modèles théoriques d'écoulement dans les corps poreux," Bulletin Rilem 29, 37 (1965)] and the BurkePlummer model [R. B. Bird, W. E. Stewart, and E. N. Lightfoot, Transport Phenomena (Wiley, New York, 2002)], with the model coefficients determined from simulations reported by W. P. Breugem, B. J. Boersma, and R. E. Uittenbogaard ["Direct numerical simulation of plane channel flow over a 3D Cartesian grid of cubes," Proceedings of the Second International Conference on Applications of Porous Media, edited by A. H. Reis and A. F. Miguel (Évora Geophysics Center, Évora, 2004), p. 27]. The results of the DNS with the grid of cubes and the second DNS in which the continuum approach is used, agree very well. (C) 2005 American Institute of Physics. [DOI: 10.1063/1.1835771]
\end{abstract}

\section{INTRODUCTION}

In this research we consider turbulent channel flow over a three-dimensional (3D) Cartesian grid of cubes, where the cubes mimic a permeable wall. A permeable wall is defined here as a rigid porous wall with interconnected pores through which fluid may flow, which in our case is coupled to the flow in the channel. The study of flows over permeable walls has various applications. Examples are oil wells, heat exchangers of open-cell metal foam, and riverbeds of for instance sand or stones. To some extent, also forests and densely builtup urban areas can be considered as porous media. In most applications the structure of the porous medium is very complex and often the geometry is not known in full detail. Furthermore, the flow inside a porous medium exhibits usually a wide range of length scales. The smallest scales are typically of the order of the pore size or the diameter of the solid obstacles of which the porous medium is composed, whereas the largest scales might be of the order of the dimensions of the porous medium. The complex structure of porous media and the wide range of length scales of the flow, hampers the direct simulation of turbulent flow over and through porous media. A direct numerical simulation (DNS) in which the complete flow field is resolved above the per- meable wall as well as within the pores of the wall, would require an enormous computational power. The literature, however, provides two other methods to simulate the turbulent flow over and through a permeable wall, which are more attractive from a computational point of view.

In the first method, the effect of wall permeability is incorporated in the boundary conditions specified at the wall interface. The main advantage of this approach is that the flow inside the permeable wall need not be calculated. This approach was adopted by, e.g., Hahn et al., ${ }^{1}$ who performed DNS of turbulent flow in a plane channel with a solid top wall and a lower permeable wall. The boundary conditions used, were an extension to the boundary conditions proposed by Beavers and Joseph ${ }^{2}$ (later on referred to as BJ) to model laminar flow parallel to a permeable wall:

$$
\begin{aligned}
& \boldsymbol{\nabla} \mathbf{u} \cdot \mathbf{n}=\frac{\alpha}{\sqrt{K}}\left(\mathbf{u}-\mathbf{U}_{d}\right), \\
& \mathbf{u} \times \mathbf{n}=\mathbf{0},
\end{aligned}
$$

where $\mathbf{n}$ is the normal unit vector at the wall, $\alpha$ is an empirical coefficient of order unity, and $K$ is the permeability. The Darcy velocity $\mathbf{U}_{d}$ is given by Darcy's Law: ${ }^{3}$ 


$$
\mathbf{U}_{d}=-\frac{K}{\mu} \nabla p,
$$

where $\mu$ is the dynamic viscosity and $\nabla p$ the imposed pressure gradient. Theoretical support for the BJ conditions was given by Saffman, ${ }^{4}$ who showed that these conditions can be derived from the assumption of Stokes flow inside as well as above the permeable wall. The BJ conditions can therefore be applied to the case of turbulent channel flow only when close to the permeable wall a viscous sublayer exists, in which the flow is governed by the Stokes equations. As pointed out by Hahn et al., ${ }^{1}$ this requirement is satisfied when $\sqrt{K}$ is small compared to the viscous length scale $\nu / u_{\tau}$, where $\nu \equiv \mu / \rho$ is the kinematic viscosity, $\rho$ the mass density, $u_{\tau} \equiv \sqrt{\tau_{w}} / \rho$ the friction velocity, and $\tau_{w}$ the wall shear stress. The ratio of these two lengthscales yields the permeability Reynolds number $\operatorname{Re}_{K} \equiv \sqrt{K} u_{\tau} / \nu$, which expresses basically the ratio of the effective pore diameter $\sqrt{K}$ to the characteristic lengthscales of near-wall eddies $\nu / u_{\tau}$. For small values of $\mathrm{Re}_{K}$ the eddies are blocked by the wall and consequently a viscous sublayer exists. For large values of $\mathrm{Re}_{K}$ the eddies may penetrate the permeable wall, and consequently the formation of a viscous sublayer is prohibited. Although it cannot be fully excluded, it is not likely that the penetration of turbulence in this case can be modeled in a simple manner by means of boundary conditions, because of the complexity of the dynamics and structure of the turbulent flow near the permeable wall. Therefore, for an accurate simulation of the flow field for large $\mathrm{Re}_{K}$, we have to describe the flow inside the permeable wall as well.

The second method to simulate flows over porous media, is to model the flow inside the permeable wall as a continuum, which is coupled with the flow outside the wall. ${ }^{5}$ The theoretical basis for this continuum approach is provided by the volume-averaging method. ${ }^{6}$ In this method the flow is averaged over a small spatial volume such that the volumeaveraged flow is defined in the fluid as well as in the solid phase. The volume-averaged flow is governed by the volume-averaged Navier-Stokes (VANS) equations. These equations are similar to the equations used in large-eddy simulations (LES), ${ }^{7}$ where the difference is due to the fact that the VANS equations have to account also for the drag force that the flow through a porous medium experiences. To solve the VANS equations, closures must be provided for the subfilter-scale stress and the drag force in terms of volumeaveraged quantities. In many porous media subfilter-scale dispersion can be neglected with respect to the drag force and/or turbulent diffusion by large-scale motions. ${ }^{5}$ Breugem $^{5}$ used in his simulations the semiempirical Ergun equation for packed beds ${ }^{8}$ to model the drag force.

A polynomial function for the porosity was adopted to model the variation of the porosity in a thin interface region in between the channel and the porous medium.

Whether the continuum approach is successful in accurately modeling the flow, depends on the accuracy of the closures for the subfilter-scale stress and especially the drag force. This motivated the present research, in which we want to evaluate these closures. To this purpose we performed a DNS of turbulent flow in a plane channel over a simple po- (a)

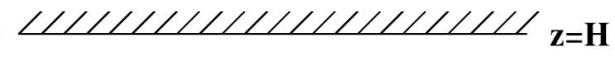

\section{mean flow direction}

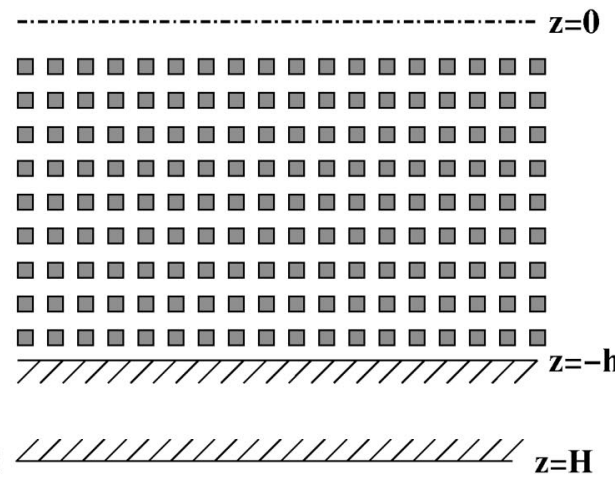

homogeneous fluid region $\varepsilon=1$

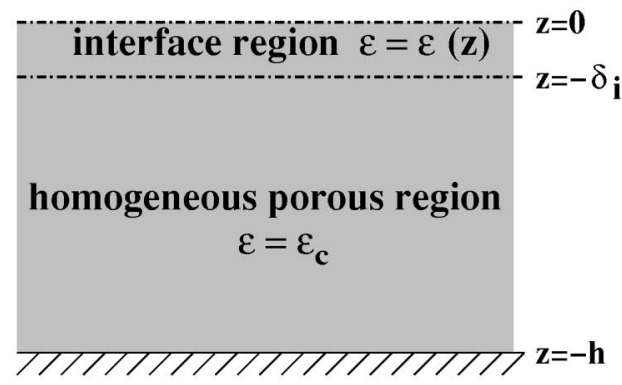

FIG. 1. Flow geometry. (a) Geometry as considered in the DNS of turbulent channel flow over a 3D Cartesian grid of cubes; (b) geometry as considered in the DNS in which a continuum approach is employed for the grid of cubes.

rous medium consisting of a 3D Cartesian grid of cubes, see Fig. 1(a). In this simulation the complete flow field in between the cubes as well as in the channel is resolved. The results of this direct simulation will be compared with a simulation in which the continuum approach is applied to the grid of cubes, see Fig. 1(b).

From now on, the DNS of the channel flow over the 3D Cartesian grid of cubes will be referred to as the DNS with cubes, whereas the DNS in which the continuum approach is employed, will be referred to as the DNS with continuum. The results from the two simulations will be compared also with a DNS of turbulent flow in a channel with two solid walls, which will be referred to as the DNS with solid walls.

This paper is organized as follows. The choice for the grid of cubes as a model porous medium is motivated in Sec. II. In Sec. III the VANS equations are introduced. In Sec. IV 


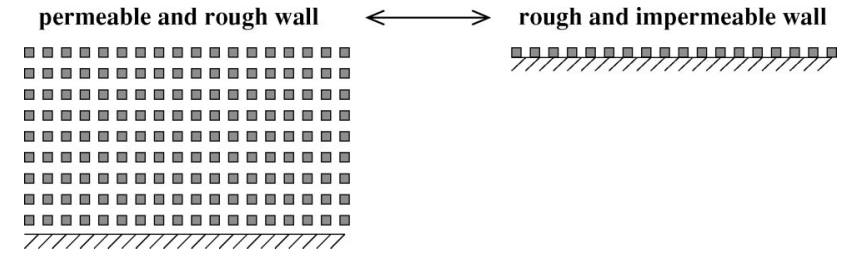

FIG. 2. Illustration of the difference between a permeable and rough wall, and a rough and impermeable wall with the same surface roughness.

the DNS with continuum is discussed. Section V concerns the numerical method used in the DNS with cubes. In Sec. VI the results of both type of simulations are compared with each other and with the results from the DNS with solid walls. Section VII contains a summary and a discussion.

\section{CARTESIAN GRID OF CUBES}

The choice for a 3D Cartesian grid of cubes as a model geometry for a porous medium is motivated by four considerations:

(1) The grid of cubes is spherically isotropic, ${ }^{9}$ which means that the permeability tensor is isotropic: $\mathbf{K}=K \mathbf{I}$, where $\mathbf{I}$ is the unit tensor. In a volume-averaged sense the grid of cubes can therefore be considered as a simple porous medium.

(2) The grid of cubes can be classified as an ordered porous medium, which is characterized by a unit cell that can be extended periodically in space. For this type of porous medium, Quintard and Whitaker ${ }^{10}$ have proposed a dedicated filter for the volume averaging of the flow field, which will be used in the present study. The length of this filter is of the same order as the dimension of the pores and solid obstacles. Therefore, the spatial structure of the unfiltered flow field is preserved as much as possible in the volume-averaged flow field. This is a major benefit of ordered porous media over disordered porous media, because for the latter the filter length should be significantly larger for averaging out local inhomogeneities in the flow field. ${ }^{10}$

(3) Permeable walls are not only permeable, but exhibit also surface roughness. In principle, the additional effect of surface roughness could be quantified by comparing the flow over a permeable and rough wall with the flow over a rough and impermeable wall with the same surface roughness. The latter wall is impermeable in the sense that below the geometrical roughness height the flow is forced to zero. The difference between the two walls is illustrated in Fig. 2. In the literature it is common practice to characterize a rough wall by the typical height of the roughness elements, which in our case is the cube rib $d_{p}$. The effect of roughness depends on the roughness Reynolds number $\operatorname{Re}_{d} \equiv d_{p} u_{\tau} / \nu$. ${ }^{11}$ Similarly, the effect of permeability depends on the permeability Reynolds number $\mathrm{Re}_{K}{ }^{5}$, which we have already introduced. Our research interest is on the influence of wall permeability rather than the effect of wall roughness on turbulent channel flow. This demands that the roughness Reynolds number is sufficiently small and that the per- meability Reynolds number is sufficiently large. The cubes are therefore aligned in a Cartesian grid and the cube rib $d_{p}$ is chosen small compared to the channel height. Furthermore, the cube rib is chosen equal to the pore dimension $\left(d_{p}=d_{f}\right)$ to ensure a relatively high permeability Reynolds number. Besides, the choice for $d_{p}$ $=d_{f}$ seems intuitively also a good choice from a computational point of view, because both the flow along and the flow in between the cubes must be resolved.

(4) The cubes can be aligned along a Cartesian computational mesh. This simplifies the volume averaging of the simulation data. An immersed boundary method (IBM) (Ref. 12 and references therein) is employed in which forces are added to the flow field to enforce the no-slip and no-penetration conditions on the cubes. This method allows for the application of fast and accurate numerical algorithms.

\section{THE VANS EQUATIONS}

In this section a brief derivation of the VANS equations is given, based on local volume averaging of the NavierStokes equations.

The formal definition of the volume-averaged velocity at position $\mathbf{x}$ is given by

$$
\langle\mathbf{u}\rangle_{\mathbf{x}}^{s} \equiv \int_{V} \gamma(\mathbf{r}) m(\mathbf{y}) \mathbf{u}(\mathbf{r}) d V,
$$

where the brackets $\langle. .\rangle^{s}$ denote the superficial volume average, the subscript $\mathbf{x}$ means that the volume average is evaluated at position $\mathbf{x}, \mathbf{y}=\mathbf{r}-\mathbf{x}$ is the position vector relative to the centroid $\mathbf{x}$ of the averaging volume $V, \gamma$ is the phaseindicator function that equals unity when $\mathbf{r}$ points into the fluid phase and that equals zero when $\mathbf{r}$ points into the solid phase, and $m$ is a weighting function. The volume-averaging operator acts as a filter, which passes only information on the large-scale structure of the flow field. Furthermore, we note that the volume-averaged flow field is continuous in the sense that it is defined both in the fluid and the solid phase, provided of course that the filter length or the averaging volume is large enough. This is the basis of the continuum approach for flow in porous media.

For a meaningful definition of volume-averaged quantities, the weighting function must satisfy the following normalization condition:

$$
\int_{V} m(\mathbf{y}) d V=1 .
$$

In principle the weighting function can be chosen freely, but it is desirable that the volume-averaged flow field contains negligible variations on scales smaller than the averaging volume; and the averaging volume is small in order to preserve as much information of the unfiltered flow field as possible in the volume-averaged flow field.

These two conditions demand that the weighting function matches the topology of the porous medium. With this in mind, Quintard and Whitaker ${ }^{10}$ proposed the following weighting function for an ordered porous medium: 
(a)

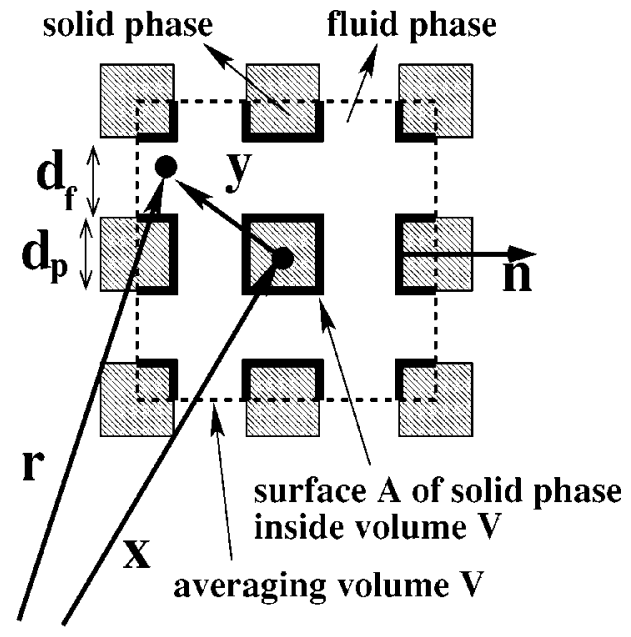

(b)

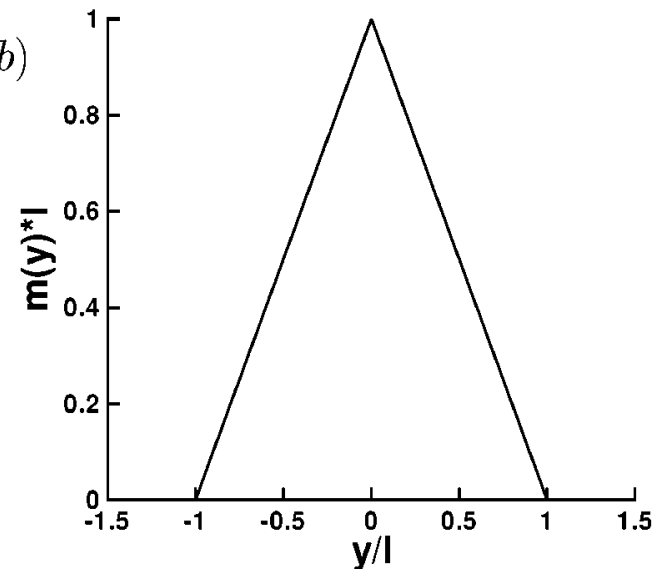

FIG. 3. The averaging volume (a) and the weighting function (b) corresponding to the cellular filter for flow in an ordered porous medium.

$$
m(\mathbf{x})=\prod_{i=1}^{3} \frac{G\left(x_{i}, l_{i}\right)}{l_{i}^{2}},
$$

in which $l_{i}$ and $x_{i}$ are, respectively, the length of the unit cell and the spatial coordinate in direction $i$. The function $G$ is defined according to

$$
G\left(x_{i}, l_{i}\right) \equiv\left\{\begin{array}{l}
l_{i}-\left|x_{i}\right|, \quad\left|x_{i}\right| \leqslant l_{i}, \\
0, \quad\left|x_{i}\right|>l_{i} .
\end{array}\right.
$$

The above averaging volume and the weighting function are illustrated in Fig. 3 for the Cartesian grid of cubes considered in the present study. Quintard and Whitaker refer to this filter as the cellular filter.

According to Eq. (4) the porosity is defined as

$$
\epsilon(\mathbf{x}) \equiv \int_{V} \gamma(\mathbf{r}) m(\mathbf{y}) d V
$$

The velocity at a certain point in the fluid phase of the porous medium can be decomposed into a contribution of the volume-averaged velocity at this point and a subfilter-scale velocity $\widetilde{\mathbf{u}}{ }^{13}$

$$
\mathbf{u}=\langle\mathbf{u}\rangle+\widetilde{\mathbf{u}},
$$

where $\langle\mathbf{u}\rangle \equiv\langle\mathbf{u}\rangle^{s} / \epsilon$ is known as the intrinsic volume- averaged velocity. Besides the spatial decomposition given by (9), in this study also a temporal decomposition will be used:

$$
\mathbf{u}=\overline{\mathbf{u}}+\mathbf{u}^{\prime},
$$

where the overbar denotes the Reynolds- or ensembleaveraged value and the prime denotes the temporal fluctuation. The spatial and temporal decompositions can be combined together, which yields

$$
\mathbf{u}=\overline{\langle\mathbf{u}\rangle}+\langle\mathbf{u}\rangle^{\prime}+\overline{\widetilde{\mathbf{u}}}+\widetilde{\mathbf{u}}^{\prime} .
$$

It is easy to show that the Reynolds- and spatial-averaging operators commute: ${ }^{14} \overline{\langle\mathbf{u}\rangle}=\langle\overline{\mathbf{u}}\rangle,\langle\mathbf{u}\rangle^{\prime}=\left\langle\mathbf{u}^{\prime}\right\rangle, \overline{\widetilde{\mathbf{u}}}=\tilde{\overline{\mathbf{u}}}$, and $\widetilde{\mathbf{u}}^{\prime}=\widetilde{\mathbf{u}}^{\prime}$.

For deriving the volume-averaged form of the NavierStokes equations it is necessary to relate the volume average of a spatial derivative to the spatial derivative of a volume average. This relation is given by the spatial-averaging theorem. ${ }^{15}$ For instance, the volume average of the gradient of the pressure is given by

$$
\langle\boldsymbol{\nabla} p\rangle_{\mathbf{x}}^{s}=\boldsymbol{\nabla}\langle p\rangle_{\mathbf{x}}^{s}+\int_{A} m(\mathbf{y}) \mathbf{n} p(\mathbf{r}) d A,
$$

where $A$ is the contact area between the fluid and the solid phase inside the averaging volume $V$, see Fig. 3(a), and $\mathbf{n}$ is the normal unit vector at $A$ that points from the fluid into the solid phase. The single assumption in the derivation of (8) is that the weighting function $m$ depends only on $\mathbf{y}$ and not on $\mathbf{x}$.

The application of the volume-averaging operator (4) and the spatial-averaging theorem (12) to the Navier-Stokes equations yields the VANS equations: ${ }^{16}$

$$
\begin{aligned}
\frac{\partial\langle\mathbf{u}\rangle^{s}}{\partial t} & +\boldsymbol{\nabla}\left[\frac{\langle\mathbf{u}\rangle^{s}\langle\mathbf{u}\rangle^{s}}{\epsilon}\right]+\boldsymbol{\nabla}\left[\langle\mathbf{u u}\rangle^{s}-\frac{\langle\mathbf{u}\rangle^{s}\langle\mathbf{u}\rangle^{s}}{\epsilon}\right] \\
& =-\frac{1}{\rho} \boldsymbol{\nabla}\langle p\rangle^{s}+\nu \nabla^{2}\langle\mathbf{u}\rangle^{s}+\int_{A} m \mathbf{n}\left[-\mathbf{I} \frac{p}{\rho}+\nu \boldsymbol{\nabla} \mathbf{u}\right] d A,
\end{aligned}
$$

$\boldsymbol{\nabla} \cdot\langle\mathbf{u}\rangle^{s}=0$.

The VANS equations as given above are exact for Newtonian and incompressible flow through a rigid porous medium. The third term on the left-hand side of Eq. (13) represents subfilter-scale dispersion, i.e., dispersion of volume-averaged momentum by subfilter-scale motions, and the last term on the right-hand side accounts for the drag force that the solid phase exerts on the fluid phase.

Darcy's Law (3) follows from the VANS equations for uniform, stationary flow through a homogeneous porous medium (i.e., with a constant porosity):

$$
0=-\boldsymbol{\nabla}\langle p\rangle-\mu \mathbf{K}^{-1}\langle\mathbf{u}\rangle^{s},
$$

where the surface integral has been replaced by $-\nu \mathbf{K}^{-1} \boldsymbol{\epsilon}\langle\mathbf{u}\rangle^{s}{ }^{16}$ The VANS equations can be considered as a generalization of the LES equations as well. The latter follow from the VANS equations for the case that the porosity equals unity, i.e., the absence of a solid phase, and consequently a zero drag force. 


\section{DNS WITH CONTINUUM}

As depicted in Fig. 1(b), in the DNS with continuum the flow domain is divided into three regions.

(1) The homogeneous fluid region or channel region between $z=0$ and $z=H$ in which the porosity $\epsilon$ equals unity.

(2) The interface region between $z=-\delta_{i}$ and $z=0$, which is characterized by a spatially varying porosity.

(3) The homogeneous porous region between $z=-h$ and $z$ $=-\delta_{i}$, with a constant porosity $\left(\epsilon=\epsilon_{c}\right)$.

Below we discuss how the flow in each region is described.

\section{A. Homogeneous porous region}

The volume-averaged flow in the homogeneous porous region is governed by the VANS equations (13) and (14). These equations can be simplified by assuming that the volume-averaged flow field does not vary significantly inside the averaging volume, i.e., $\langle\langle\mathbf{u}\rangle\rangle \approx\langle\mathbf{u}\rangle$. This is equivalent to the assumption that $\langle\tilde{\mathbf{u}}\rangle \approx \mathbf{0}$. If this assumption holds, then Eq. (13) can be written as ${ }^{10}$

$$
\begin{aligned}
\frac{\partial\langle\mathbf{u}\rangle^{s}}{\partial t} & +\nabla\left[\frac{\langle\mathbf{u}\rangle^{s}\langle\mathbf{u}\rangle^{s}}{\epsilon}\right]+\nabla\langle\widetilde{\mathbf{u}}\rangle^{s} \\
= & -\frac{\boldsymbol{\epsilon}}{\rho} \boldsymbol{\nabla} \frac{\langle p\rangle^{s}}{\epsilon}+\nu \nabla^{2}\langle\mathbf{u}\rangle^{s}-\nu \nabla \boldsymbol{\epsilon} \cdot \boldsymbol{\nabla} \frac{\langle\mathbf{u}\rangle^{s}}{\boldsymbol{\epsilon}} \\
& +\int_{A} m \mathbf{n}\left[-\mathbf{I} \frac{\tilde{p}}{\rho}+\nu \nabla \widetilde{\mathbf{u}}\right] d A .
\end{aligned}
$$

In order to solve the above equations, closures are required for the subfilter-scale stress and the drag force in terms of the volume-averaged flow field. Whitaker ${ }^{16}$ gave support to the following convenient parametrization of the surface integral in Eq. (16):

$$
\int_{A} m \mathbf{n}\left[-\mathbf{I} \frac{\tilde{p}}{\rho}+\nu \boldsymbol{\nabla} \widetilde{\mathbf{u}}\right] d A=-\nu \mathbf{K}^{-1}(\mathbf{I}+\mathbf{F}) \boldsymbol{\epsilon}\langle\mathbf{u}\rangle^{s},
$$

where $\mathbf{K}, \mathbf{I}$ and $\mathbf{F}$ are, respectively, the permeability, the unit, and the Forchheimer tensor. A 3D Cartesian grid of cubes falls in the class of spherically isotropic porous media ${ }^{9}$ for which the permeability tensor is isotropic: $\mathbf{K}=K \mathbf{I}$. Irmay ${ }^{17}$ derived the following expression for the permeability $K$ of the grid of cubes, valid for $\epsilon \ll 1$ :

$$
\mathbf{K}=K \mathbf{I}, \quad K=\frac{\left[1-(1-\epsilon)^{1 / 3}\right]^{3}\left[1+(1-\epsilon)^{1 / 3}\right]}{C_{K}(1-\epsilon)} d_{p}^{2},
$$

where $C_{K}=12$ on condition that $\epsilon \ll 1$ holds. In general, the Forchheimer tensor $\mathbf{F}$ depends on the Reynolds number, on the geometrical parameters of the porous medium and on the direction of the volume-averaged flow. In the present work, $\mathbf{F}$ is modeled with the Burke-Plummer equation, which is equivalent to the nonlinear part of the Ergun equation: ${ }^{8}$

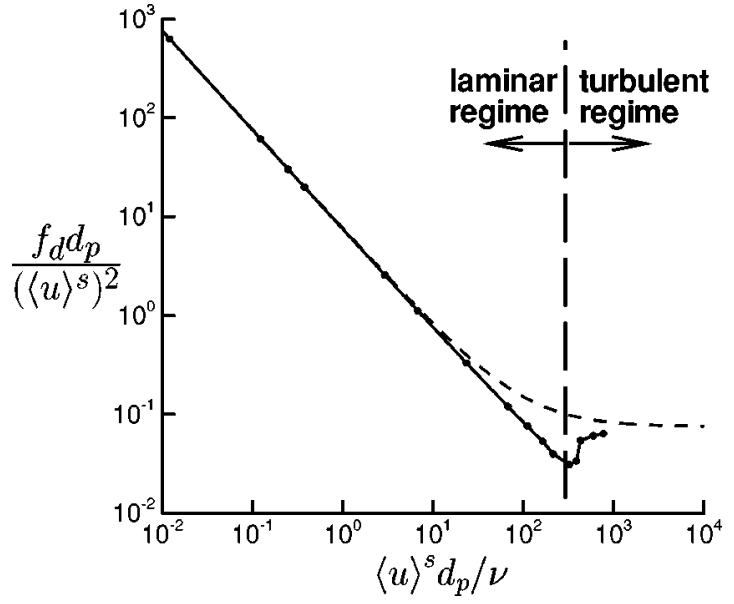

FIG. 4. Drag force $f_{d}=(1 / \epsilon) \int_{A} m \mathbf{n} \cdot[-\mathbf{I} \hat{p} / \rho+\nu \nabla \widetilde{\mathbf{u}}] \cdot \mathbf{n}_{x} d A$ per unit mass of the fluid phase as function of the Reynolds number, where $\mathbf{n}_{x}$ is the unit vector in the streamwise direction. Each dot corresponds to a separate simulation (Ref. 19). The simulation results are compared with a model based on Eqs. (17)-(19) with $C_{K}=11.4$ and $C_{F}=0.4$. —, Linear interpolation of simulation results; ---, model.

$$
\mathbf{F}=F \mathbf{I}, \quad \frac{d_{p}^{2} F}{K}=C_{F}\left(\frac{1-\epsilon}{\epsilon^{3}}\right)\left(\frac{\left|\langle\mathbf{u}\rangle^{s}\right| d_{p}}{\nu}\right),
$$

where $C_{F}$ is a constant and for many packed beds approximately equal to $1.8 .^{18}$ In order to determine the values of $C_{K}$ and $C_{F}$ more accurately, Breugem et al. ${ }^{19}$ performed detailed numerical simulations of flow through a fully periodic 3D Cartesian grid of cubes with $\epsilon=0.875$ (which is equivalent to $d_{f}=d_{p}$ ). Based on these simulations we found that $C_{K}=11.4$ and that $C_{F} \approx 0.4$. The results from these simulations, together with the model prediction based on equations (17)-(19), are depicted in Fig. 4. The model overestimates the drag force in the transitional regime where both linear and nonlinear drag are important, but gives a good fit in the low and the high Reynolds number range.

A discussion of the closure problem for the subfilterscale stress $\tau \approx\langle\widetilde{\mathbf{u}} \widetilde{\mathbf{u}}\rangle^{s}$ is given by Breugem. ${ }^{5}$ It is argued that in porous media subfilter-scale dispersion is usually negligible with respect to the drag force and/or the Reynoldsshear stress of the volume-averaged flow field. The final form of the momentum equation as used in the continuum approach, therefore reads

$$
\begin{aligned}
\frac{\partial\langle\mathbf{u}\rangle^{s}}{\partial t} & +\nabla\left[\frac{\langle\mathbf{u}\rangle^{s}\langle\mathbf{u}\rangle^{s}}{\epsilon}\right] \\
= & -\frac{\epsilon}{\rho} \nabla \frac{\langle p\rangle^{s}}{\epsilon}+\nu \nabla^{2}\langle\mathbf{u}\rangle^{s}-\nu \boldsymbol{\nabla} \epsilon \cdot \nabla \frac{\langle\mathbf{u}\rangle^{s}}{\epsilon} \\
& -\frac{\nu}{K}(1+F) \epsilon\langle\mathbf{u}\rangle^{s},
\end{aligned}
$$

where $K$ and $F$ are given by, respectively, Eqs. (18) and (19).

\section{B. Channel region}

The VANS equations (13) and (14) apply to the channel region as well. Because the porosity equals unity in this region, the drag term on the right-hand side of (13) vanishes 
and hence the VANS equations reduce to the LES equations. The subfilter-scale dispersion term is negligible only when the filter length is sufficiently small. This may not be the case when the same filter length is used for the channel region as for the homogeneous porous region. In the DNS with continuum, however, we aim to completely resolve the flow field in the channel region. This can be accomplished by assuming that in the channel region the filter length is set by the computational mesh spacing $\Delta$, such that, provided that $\Delta$ is sufficiently small, dispersion by subfilter-scale turbulent motions is negligible. Consequently, the VANS equations (13) and (14) reduce to the standard Navier-Stokes equations with $\langle\mathbf{u}\rangle^{s}=\mathbf{u}$.

\section{Interface region}

As pointed out above, we assume that in the DNS with continuum the cellular-filter length is different for the homogeneous porous region than for the channel region. This implies that the filter length varies across the interface region, with $l_{f}=d_{f}+d_{p}$ (see Fig. 3) in the homogeneous porous region and $l_{f}=\Delta$ in the channel region. Consequently, in the interface region the volume-averaging theorem (12) is not strictly valid, because a spatially varying filter length means that the weighting function $m$ depends not only on $\mathbf{y}$, but also on $\mathbf{x}$. On the other hand, the commutation errors will be small when the filter length is gradually changed over a sufficiently large distance compared to the characteristic length scales of the flow field. ${ }^{20}$ The VANS equations (13) and (14) may therefore be applied also to the interface region. As for the homogeneous porous region and the channel region, we assume that subfilter-scale dispersion can be neglected in the interface region too. The drag force cannot be neglected, however. It is modeled by means of Eq. (17), with Eqs. (18) and (19) for $K$ and $F$, respectively. Furthermore, we need a model for the porosity, which must gradually change across the interface region from unity at $z=0$ to $\epsilon=\epsilon_{c}$ at $z=-\delta_{i}$. In a previous study ${ }^{5}$ we adopted a fifth-order polynomial for $\epsilon$ according to

$$
\begin{aligned}
-\delta_{i} \leqslant z \leqslant 0: \epsilon(z)= & -6\left(\epsilon_{c}-1\right)\left(\frac{z}{\delta_{i}}\right)^{5}-15\left(\epsilon_{c}-1\right)\left(\frac{z}{\delta_{i}}\right)^{4} \\
& -10\left(\epsilon_{c}-1\right)\left(\frac{z}{\delta_{i}}\right)^{3}+1
\end{aligned}
$$

This model requires a specification of the thickness $\delta_{i}$. If the length of the cellular filter would be kept constant at $l_{f}=d_{p}$ $+d_{f}$, then $\delta_{i}=3 d_{p}$ for the 3D grid of cubes with $d_{f}=d_{p}$. The corresponding porosity profile is plotted in Fig. 5 together with the porosity profile calculated from Eq. (8). The agreement between the model and the exact porosity profile is good. The local value for the porosity in the interface region is used in the expressions for $K$ and $F$. This assures that the drag force and the VANS equations are continuous across the interface region. The reader may notice that the permeability approaches infinity at $z=0$. However, in order to solve Eq. (20), we actually compute $1 / K$, which remains bounded throughout the flow domain.

Recapitulating, in the DNS with continuum, Eqs. (14)

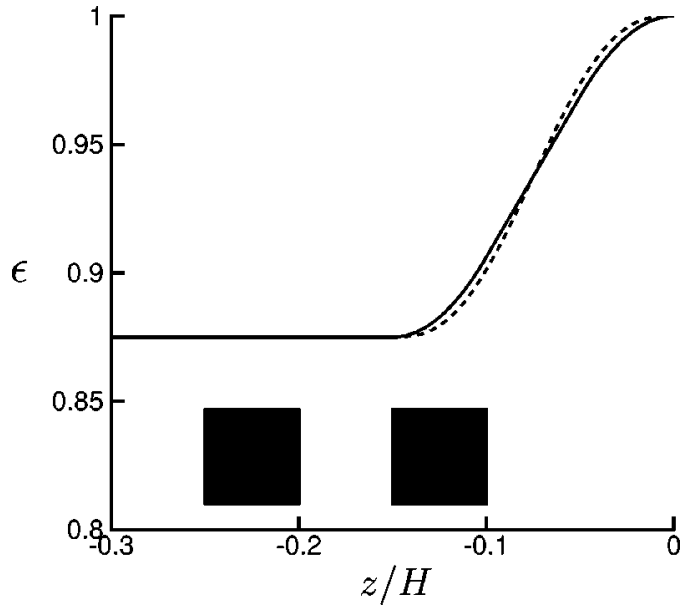

FIG. 5. Porosity profile corresponding to the flow over a 3D Cartesian grid of cubes with $d_{f}=d_{p}$ and $\delta_{i}=3 d_{p}$. The black squares mark the positions of the cubes. - , Calculated from Eq. (8); ---, calculated from Eq. (21).

and (20) are solved simultaneously for both the channel and the porous medium. The porosity in the interface region is given by Eq. (21). The permeability and the Forchheimer parameter are calculated from, respectively, Eqs. (18) and (19). The governing equations are solved by means of a second-order pressure-correction method. A pseudospectral method is used for the spatial derivatives in the wall-parallel directions, whereas the finite-volume method with the central-differencing scheme is used for the wall-normal direction. The reader is referred to Breugem ${ }^{5}$ for more details on the numerical scheme. The same code has also been used for the DNS with solid walls. The results from the latter simulation are in excellent agreement with the DNS results of Kim et al. ${ }^{21}$

\section{DNS WITH CUBES}

In this section the numerical method is discussed that is used in the DNS with cubes. The flow geometry has been presented in Fig. 1(a). The computational domain has finite dimensions. It is bounded by two solid walls at $z=H$ and $z$ $=-h$, respectively, at which the no-slip and no-penetration boundary conditions are imposed. Periodic boundary conditions are imposed for the wall-parallel directions. The Navier-Stokes equations are discretized on a fully staggered and uniform Cartesian mesh by means of the finite-volume method based on the second-order central-differencing scheme. The equations are advanced in time with the following pressure-correction scheme:

$$
\begin{aligned}
& \frac{\hat{u}_{i}-u_{i}^{n}}{\Delta t}=\frac{5}{4} g_{i}^{n}-\frac{1}{4} g_{i}^{n-2}-\frac{1}{\rho} \frac{d P}{d x} \delta_{i 1}, \\
& \frac{1}{\rho} \frac{\partial^{2} \hat{p}}{\partial x_{i}^{2}}=\frac{1}{\Delta t} \frac{\partial \hat{u}_{i}}{\partial x_{i}}, \\
& u_{i}^{n+1}=\hat{u}_{i}-\frac{\Delta t}{\rho} \frac{\partial \hat{p}}{\partial x_{i}},
\end{aligned}
$$




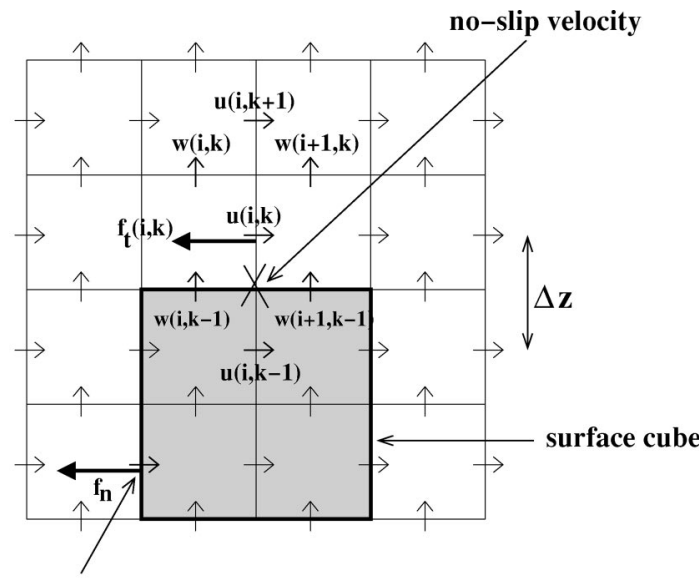

very small penetration velocity

FIG. 6. Illustration of the IBM in which forces (indicated by $f_{t}$ and $f_{n}$ ) are applied at mesh points close to and at the surface of the cube to enforce the no-slip and no-penetration conditions. The horizontal and vertical vectors mark the mesh points of the streamwise and wall-normal velocity components, respectively.

$$
p^{n+1}=p^{n}+\hat{p},
$$

where $\hat{u}_{i}$ is the prediction velocity, $d P / d x$ the constant pressure gradient that drives the flow, and $\hat{p}$ is the correction pressure. The function $g_{i}$ in Eq. (22) is given by

$$
g_{i}=-\frac{1}{\rho} \frac{\partial p}{\partial x_{i}}-\frac{\partial u_{i} u_{j}}{\partial x_{j}}+\nu \frac{\partial^{2} u_{i}}{\partial x_{j}^{2}}+f_{i} .
$$

For the implementation of the cubes an IBM is used, which has been used successfully by Fadlun et al., ${ }^{12}$ and references therein. In this method forces are added to the flow field, i.e., $f_{i} \neq 0$ in Eq. (26), to accomplish a zero velocity on the cubes, as illustrated in Fig. 6. The advantage of an IBM is that no boundary conditions have to be specified on the cubes. As a consequence of this, the Poisson equation (23) can be solved on a continuous domain with very efficient FFT-based solvers.

The IBM that is used here, is similar to the one developed by Fadlun et al. ${ }^{12}$ An advantage of applying the IBM in the present study over the problems discussed by Fadlun et al., which concern rather complicated geometries, is that the cubes can be aligned along the computational mesh. As sketched in Fig. 6, the cubes are aligned along the mesh such that their surfaces coincide with mesh points for the normal (with respect to the surface) velocity. This enables an exact implementation of the no-slip boundary condition on the cubes:

$$
\mathbf{u} \times \mathbf{n}=\mathbf{0} .
$$

As an example we calculate the force $f_{t}$ at position $(i, k)$ in Fig. 6 that is equivalent to imposing a no-slip velocity at the location of the cross. The discretized terms in Eq. (26) for the function $g$ that make use of the velocity components $u_{(i, k-1)}, w_{(i, k-1)}$ and $w_{(i+1, k-1)}$, are

$$
-\left.\frac{\partial u w}{\partial z}\right|_{(i, k)}=-\left[\frac{u w_{(i, k+1 / 2)}-u w_{(i, k-1 / 2)}}{\Delta z}\right] \text {, }
$$

$$
\left.\nu \frac{\partial^{2} u}{\partial z^{2}}\right|_{(i, k)}=\nu\left[\frac{u_{(i, k+1)}-2 u_{(i, k)}+u_{(i, k-1)}}{\Delta z^{2}}\right],
$$

where $u w_{(i, k-1 / 2)}$ corresponds to $u w$ at the location of the cross. According to the desired no-slip condition, $u w_{(i, k-1 / 2)}$ $=0$ and $u_{(i, k-1)}=-u_{(i, k)}$ must hold. This is equivalent to a force $f_{t}$ equal to

$$
\left.f_{t}\right|_{(i, k)}=-\frac{u w_{(i, k-1 / 2)}}{\Delta z}-\nu\left[\frac{u_{(i, k)}+u_{(i, k-1)}}{\Delta z^{2}}\right] .
$$

The no-penetration condition on the cubes is enforced by putting the prediction velocity $\hat{u}_{i}$ to zero. According to Eq. (24) this yields a penetration velocity equal to

$$
\mathbf{u}^{n+1} \cdot \mathbf{n}=-\Delta t \nabla \hat{p} \cdot \mathbf{n} \approx 0 .
$$

Because the pressure is updated every time step, see Eq. (25), $\nabla \hat{p}$ remains very small. As $\Delta t$ is also small, the righthand side of (28) is therefore almost zero. For the case of a stationary flow, the correction pressure will approach zero and the no-penetration condition is then enforced exactly. In the DNS with cubes it has been verified that the largest penetration velocities, which appeared at the corners of the cubes nearest to $z=0$ in Fig. 1(a) have a magnitude smaller than $10^{-5}$ times the bulk velocity in the channel.

In the IBM used in the DNS with cubes, forces are not only applied at the outside of the cubes, but also at the inside. Furthermore, the pressure gradient $d P / d x$ on the righthand side in Eq. (22) is set to zero in the interior of the cubes. The interior of a cube is therefore very similar to a closed cavity inside which the "fluid" is at rest.

The use of the IBM has consequences for the stability of the numerical scheme. The forces that are added to the momentum equation are related to the drag that the flow encounters by the presence of the cubes. From Eq. (17) for the drag force in the VANS equations, it can be deduced that this may cause large negative eigenvalues of the function $g_{i}$. It is however difficult to quantify this in a simple manner, as Eq. (17) concerns the drag force experienced by the volumeaveraged flow, whereas $f_{i}$ in the equation for $g_{i}$ is a point force acting on the unfiltered flow field. The expected difficulties with large negative eigenvalues, motivated our choice to use another time integration scheme (22) than the popular second-order Adams-Bashforth scheme. ${ }^{22}$ The stability polynomial for our scheme is found by putting $u_{i}^{n+1}=e^{i \phi} u_{i}^{n}$ with $\phi \in[0,2 \pi)$ and solving the eigenvalue $\lambda$ from

$$
u_{i}^{n+1}-u_{i}^{n}=\lambda \Delta t\left[\frac{5}{4} u_{i}^{n}-\frac{1}{4} u_{i}^{n-2}\right] .
$$

The solution reads

$$
\lambda \Delta t=4 e^{2 i \phi}\left(\frac{e^{i \phi}-1}{5 e^{2 i \phi}-1}\right) .
$$

The above stability polynomial is shown in Fig. 7. Also depicted in this figure is the stability polynomial of the secondorder Adams-Bashforth scheme. From the figure it is clear that with respect to the Adams-Bashforth scheme, the time integration scheme used in the present study is much more stable for eigenvalues with a large negative real part. The dotted line in Fig. 7 is the stability polynomial of the 


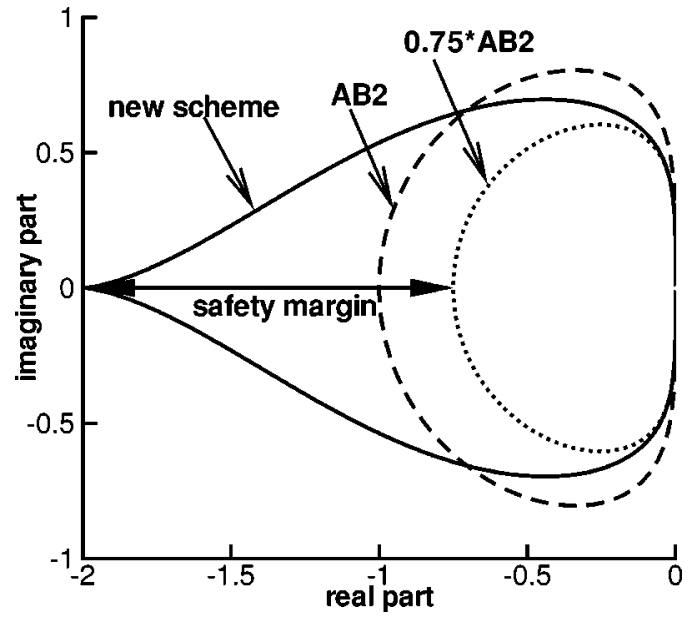

FIG. 7. Stability polynomials for several time integration schemes. The enclosed areas are the stable regions of the respective schemes. -, Eq. (30); ---, stability polynomial of second-order Adams-Bashforth scheme; ..., stability polynomial of second-order Adams-Bashforth scheme multiplied by 0.75 .

Adams-Bashforth scheme multiplied by a factor of $3 / 4$, which falls inside the curve of the stability polynomial given by (30). Ignoring the forces added to the momentum equation in the IBM, the computational time step criteria corresponding to the latter stability polynomial are the criteria derived by Wesseling ${ }^{22}$ (p. 188) for the second-order AdamsBashforth scheme multiplied by $3 / 4$ :

$$
\frac{\nu \Delta t}{\Delta x^{2}}<\frac{1}{16}
$$

$$
\frac{\Delta t \sqrt{\sum_{i} u_{i}^{2}}}{\Delta x}<\frac{1}{4},
$$

$$
\sum_{i}\left(\frac{\Delta t\left|u_{i}\right|}{\Delta x}\right)\left(\frac{\left|u_{i}\right| \Delta x}{\nu}\right)^{1 / 3}<\left(\frac{27}{32}\right)^{1 / 3}
$$

These time step criteria are used in the present simulations. As indicated in Fig. 7, a relatively large safety margin exists to account for the destabilizing effect of the IBM forces.

\section{NUMERICAL RESULTS}

In the following sections the results from the DNS with cubes and the DNS with continuum are compared with each other and with the results from the DNS with solid walls. First some characteristics of the DNS with cubes and the DNS with continuum are given.

The dimensions of the computational domain in the DNS with cubes are $3 \times 2 \times 2$ in terms of the channel height $H$ for, respectively, the streamwise, the spanwise, and the wallnormal direction. The porous medium has a height $h=H$ and consists of $30 \times 20 \times 9=5400$ cubes in, respectively, the streamwise, spanwise, and wall-normal direction. The number of cube layers in the wall-normal direction is chosen deliberately large to prevent that the flow in the channel experiences any influence of the solid wall below the grid of cubes. The cube rib equals $d_{p}=H / 20$. The dimension $d_{f}$ of the pores is equal to $d_{p}$, and consequently the porosity $\epsilon_{c}$ in the homogeneous porous region equals 0.875 . The Darcy number is given by $\mathrm{Da}_{c} \equiv K_{c} / H^{2}=3.4 \times 10^{-4}$, where $K_{c}$ is the permeability of the grid of cubes (which was determined in Sec. IV A). The number of mesh points is $600 \times 400 \times 400$ $=96 \times 10^{6}$. The bulk Reynolds number $\operatorname{Re}_{b} \equiv U_{b} H / \nu=5500$, where $U_{b}$ is the bulk velocity in the channel region. The friction Reynolds number for the top wall $\operatorname{Re}_{\tau}^{t} \equiv u_{\tau}^{t} H / \nu$ $=394$, where $u_{\tau}^{t} \equiv[-\nu \partial\langle\bar{u}\rangle / \partial z]_{z=H}^{1 / 2}$ is the friction velocity at the top wall. The friction Reynolds number for the permeable wall $\operatorname{Re}_{\tau}^{p} \equiv u_{\tau}^{p} H / \nu=669$, where $u_{\tau}^{p} \equiv\left[-\left\langle\overline{u^{\prime} w^{\prime}}\right\rangle+\nu \partial\langle\bar{u}\rangle / \partial z\right]_{z=0}^{1 / 2}$ is the friction velocity at the permeable wall. The permeability Reynolds number $\operatorname{Re}_{K} \equiv u_{\tau}^{p} \sqrt{K_{c}} / \nu$ is equal to 12.4 . Based on the classification of Breugem ${ }^{5}$ of permeable walls, the grid of cubes can therefore be considered as a highly permeable wall near which viscous effects are of minor importance. The roughness Reynolds number $\operatorname{Re}_{d} \equiv u_{\tau}^{p} d_{p} / \nu=33.4$, which according to Hinze ${ }^{11}$ is in the transitional roughness regime. The computational time step in the simulation equals $\Delta t=6.7 \times 10^{-4} H / U_{b}$. The number of instantaneous data fields used for the statistics equals 45 , spanning a total time interval of $60.5 \mathrm{H} / U_{b}$.

The values of $\epsilon_{c}, d_{p}, \delta_{i}$, and $\mathrm{Re}_{b}$ for the DNS with continuum are chosen equal to the corresponding values in the DNS with cubes. Some characteristics of these two simulations are listed in Table I. The Reynolds numbers $\operatorname{Re}_{\tau}^{p}, \operatorname{Re}_{\tau}^{t}$, $\mathrm{Re}_{K}$, and $\mathrm{Re}_{d}$ are about equal, and, because they were determined from the simulation data, this indicates already that also the turbulence statistics of the two simulations are simi-

TABLE I. Characteristics of the DNS with cubes (DNS CUB), DNS with continuum (DNS CON), and the DNS with solid walls (DNS SOL). $\epsilon_{c}$ is the porosity in the homogeneous porous region, $d_{p} / H$ is the rib of the cubes, $\mathrm{Da}_{c} \equiv K_{c} / H^{2}$ is the Darcy number in the homogeneous porous region where $K=K_{c}, \delta_{i}$ is the thickness of the interface region, $h$ is the thickness of the permeable wall, $\operatorname{Re}_{b} \equiv U_{b} H / \nu$ is the bulk Reynolds number where $U_{b}$ is the bulk velocity in the channel, $\operatorname{Re}_{\tau}^{t} \equiv u_{\tau}^{t} H / \nu$ is the friction Reynolds number based on the friction velocity $u_{\tau}^{t}$ at the top wall, $\operatorname{Re}_{\tau}^{p} \equiv u_{\tau}^{p} H / \nu$ is the friction Reynolds number based on the friction velocity $u_{\tau}^{p}$ at the permeable wall, $\mathrm{Re}_{K} \equiv u_{\tau}^{p} \sqrt{K_{c}} / \nu$ is the permeability Reynolds number based on the friction velocity $u_{\tau}^{p}$ at the permeable wall, and $\operatorname{Re}_{d} \equiv u_{\tau}^{p} d_{p} / \nu$ is the roughness Reynolds number for the permeable wall.

\begin{tabular}{cllllllllll}
\hline \hline DNS & \multicolumn{1}{c}{$\epsilon_{c}$} & $d_{p} / H$ & $\mathrm{Da}_{c}\left(10^{-4}\right)$ & $\delta_{i} / H$ & $h / H$ & $\operatorname{Re}_{b}$ & $\operatorname{Re}_{\tau}^{p}$ & $\operatorname{Re}_{\tau}^{t}$ & $\operatorname{Re}_{K}$ & $\operatorname{Re}_{d}$ \\
\hline CUB & 0.875 & 0.05 & 3.4 & 0.15 & 1 & 5500 & 669 & 394 & 12.4 & 33.4 \\
CON & 0.875 & 0.05 & 3.4 & 0.15 & 1 & 5500 & 726 & 409 & 13.5 & 36.3 \\
SOL & 0 & 0 & 0 & 0 & 0 & 5500 & 352 & 350 & 0 & 0 \\
\hline \hline
\end{tabular}


lar. The dimensions of the computational domain in the DNS with continuum are $5 \times 3 \times 2$ in terms of the channel height $H$ for, respectively, the streamwise, the spanwise, and the wall-normal direction. The number of mesh points is 256 $\times 192 \times 300=15.7 \times 10^{6}$. The mesh is stretched in the wallnormal direction with mesh points clustered around the permeable wall interface and the top wall. The computational time step in the DNS with continuum equals $\Delta t=3.1$ $\times 10^{-4} \mathrm{H} / U_{b}$. The number of instantaneous data fields for the statistics equals 96, spanning a total time interval of $58.7 \mathrm{H} / U_{b}$.

We remark that the dimensions of the computational domain in the DNS with cubes are chosen deliberately smaller than in the DNS with continuum, in order to avoid that the number of mesh points in the DNS with cubes would become excessively large. Therefore, a larger computational domain was not feasible. We note that the dimensions of the computational domain in the DNS with cubes, although relatively small, are still much larger than the minimal channel studied by Jiménez and Moin. ${ }^{23}$ Furthermore, we verified by means of a simulation of standard channel flow, using the same numerical method as used in the DNS with cubes, that a computational domain of $3 \times 2 \times 1$ yields low-order statistics that agree well with the DNS results of Kim et al. ${ }^{21}$

The codes for both the DNS with cubes and the DNS with continuum have been written in FORTRAN 77 and made parallel based on the MPI standard. The DNS with cubes was run on 100 nodes of a SGI Origin 3800 system. The DNS with continuum was run on 32 nodes of a SGI Altix 3700 system.

\section{A. Volume averaging and mean velocity profiles}

Before the results of the DNS with cubes and the DNS with continuum can be compared with each other, the velocity field of the DNS with cubes need to be filtered first according to Eq. (4) with the cellular weighting function (6). We remark that the calculation of the volume-averaged velocity at a single mesh point involves the evaluation of the discretized form of Eq. (4) over $40^{3}$ neighboring mesh points. Hence, the computation of the volume-averaged velocity at all mesh points is fairly time consuming. Figure 8 shows a cross section of the fluctuating flow field before and after filtering. The white spots in Fig. 8(a) mark the location of the cubes. The rectangle indicates the dimension of the averaging volume of the cellular filter. Figure $8(\mathrm{~b})$ shows clearly that due to filtering the subfilter-scale motions are averaged out and that the volume-averaged flow field is continuous throughout the flow domain. Figure 9 shows the effect of filtering applied to the Reynolds-averaged flow field. Furthermore, it illustrates nicely the decomposition (9) of the Reynolds-averaged flow field into the volume- and Reynolds-averaged flow field and the subfilter-scale Reynolds-averaged flow field: $\bar{u}_{i}=\left\langle\bar{u}_{i}\right\rangle+\tilde{\bar{u}}_{i}$. Notice that the volume- and Reynolds-averaged flow field $(\langle\bar{u}\rangle,\langle\bar{w}\rangle)$ is onedimensional and horizontally homogeneous, whereas the unfiltered Reynolds-averaged flow field $(\bar{u}, \bar{w})$ is threedimensional and horizontally heterogeneous. The horizontal heterogeneity in the unfiltered Reynolds-averaged flow field
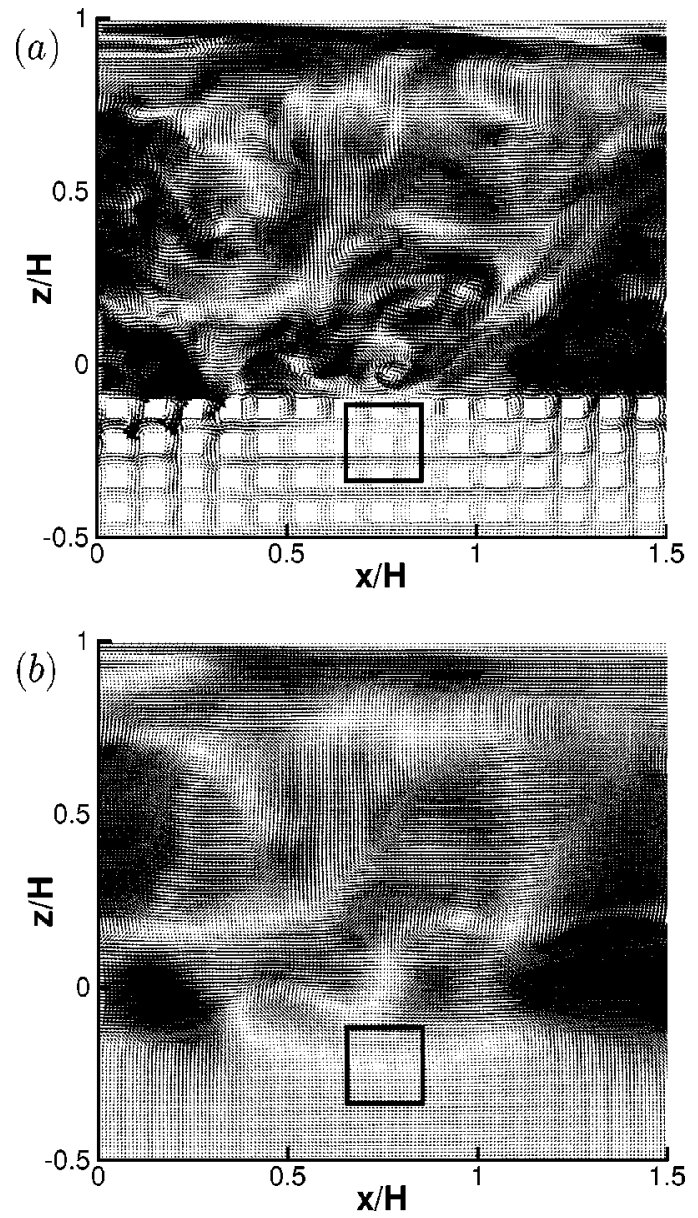

FIG. 8. Cross section along the streamwise direction of the fluctuating flow field in the DNS with cubes. For clarity, only part of the cross section is shown and the number of vectors is reduced by a factor of 2 in both the $x$ and $z$ direction. The rectangle shows the dimension of the averaging volume of the cellular filter. (a) Unfiltered flow field $\left(u^{\prime}, w^{\prime}\right)$; (b) corresponding volume-averaged flow field $\left(\left\langle u^{\prime}\right\rangle^{s},\left\langle w^{\prime}\right\rangle^{s}\right)$.

rapidly vanishes when moving out the grid of cubes into the channel region, with $|\bar{w}|_{\max } / U_{b}<10^{-2}$ for $z / H>-0.07$ and $|\bar{w}|_{\max } / U_{b}<10^{-3}$ for $z / H>0$.

It is important to note that in the DNS with cubes the filter length is kept constant at $l_{f}=d_{f}+d_{p}$ in the volume averaging of the flow field, except close to the solid walls at $z=H$ and $z=-h$ where the vertical extent of the averaging volume is gradually decreased to zero, depending on the distance to the wall. We remark that the constant filter length in the DNS with cubes is different from the assumption of a variable filter length in the DNS with continuum. Recall that the variable filter length in the latter simulation has the advantage that no subfilter-scale stress need to be modeled in the channel region. The disadvantage of a variable filter length is that it causes a commutation error in the volumeaveraging theorem (8). Although this error is small, in the DNS with cubes we want to avoid this, and therefore the filter length in the processing of this simulation is kept constant. It is important to realize that the difference in the filter length for the channel region between the DNS with cubes and the DNS with continuum, has consequences for compar- 

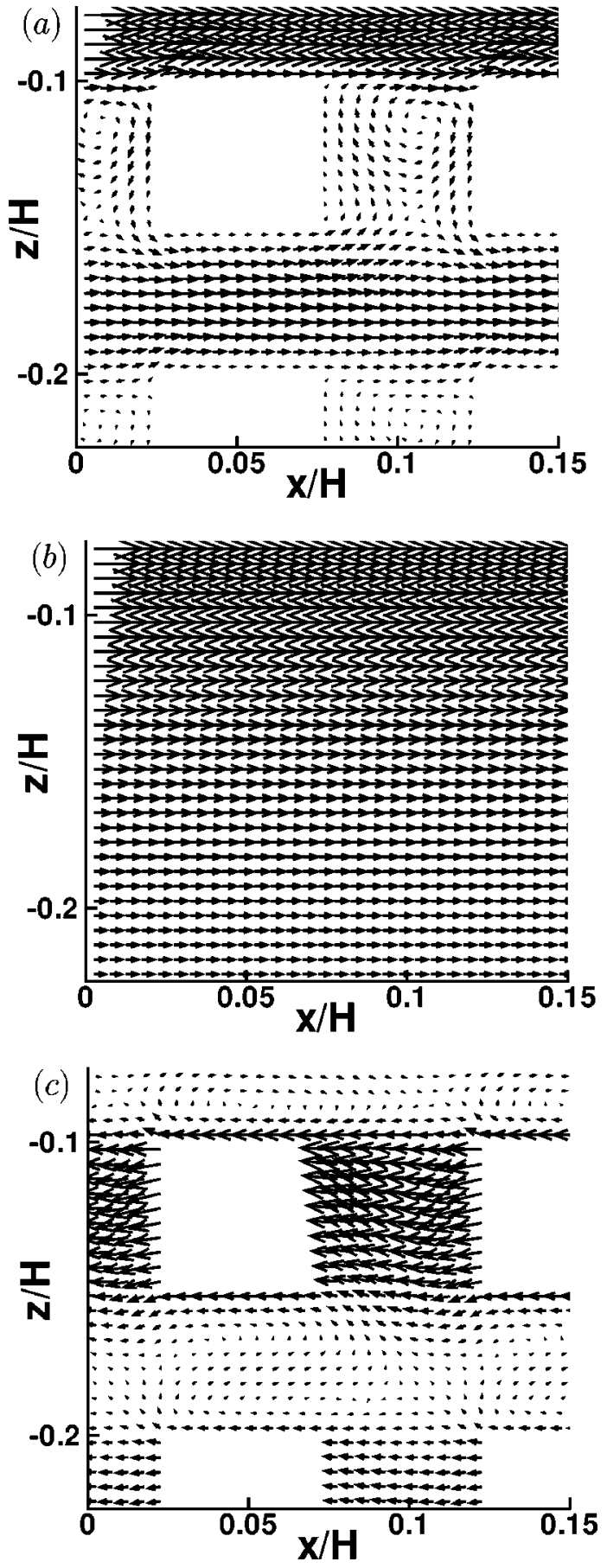

FIG. 9. Cross section along the streamwise direction of the Reynoldsaveraged flow field in the DNS with cubes. For clarity, only part of the cross section is shown. The different graphs illustrate the decomposition $\bar{u}_{i}=\left\langle\bar{u}_{i}\right\rangle$ $+\tilde{\bar{u}}_{i}$. (a) Reynolds-averaged flow field $(\bar{u}, \bar{w})$; (b) volume- and Reynoldsaveraged flow field $(\langle\bar{u}\rangle,\langle\bar{w}\rangle)$; (c) subfilter-scale Reynolds-averaged flow field $(\tilde{\bar{u}}, \tilde{\bar{w}})$.

ing the results of the two simulations with each other. We come back to this point in the next section.

Figure 10 presents the profiles of the Reynolds- and volume-averaged velocity of the DNS with cubes, the DNS with continuum and the DNS with solid walls. We recall that in the channel region of the DNS with continuum and the DNS with solid walls, the Reynolds- and volume-averaged velocity $\langle\bar{u}\rangle^{s}$ is equal to the Reynolds-averaged velocity $\bar{u}$,

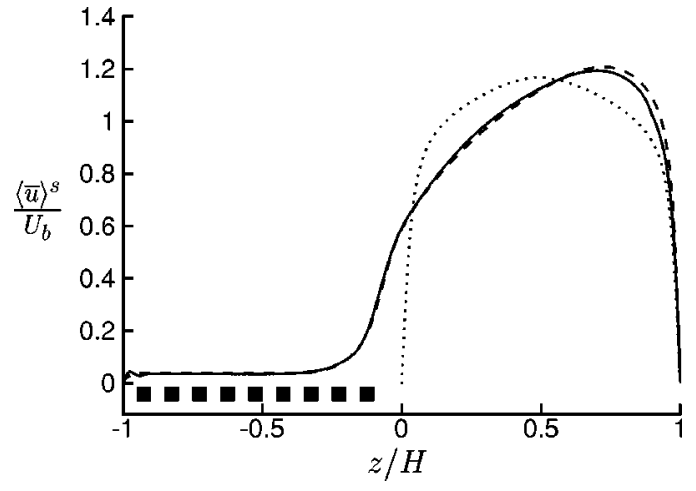

FIG. 10. Profiles of the Reynolds- and volume-averaged velocity, normalized by the bulk velocity $U_{b}$, as function of $z / H$. The black squares mark the location of the cubes in the DNS with cubes. -, DNS with cubes; --- DNS with continuum; ..., DNS with solid walls.

because the mesh spacing is sufficiently small. In the channel region of the DNS with cubes, the profile of $\langle\bar{u}\rangle^{s}$ is also very similar, although not exactly equal, to $\bar{u}$. Although our main interest is in the behavior of the flow field in the channel region, we still prefer here to plot $\langle\bar{u}\rangle^{s}$, because this velocity is continuous and horizontally homogeneous in the channel region as well as inside the permeable wall. This is not true for $\bar{u}$.

The profiles of the DNS with cubes and the DNS with continuum overlap each other. Both profiles are strongly skewed with the position $z=\delta_{w}$ of the maximum velocity well above the centerline of the channel. The position of the maximum velocity corresponds to zero total shear stress. From this condition the following expression can be found for $\delta_{w}$ :

$$
\frac{\delta_{w}}{H}=\frac{\left(u_{\tau}^{p}\right)^{2}}{\left(u_{\tau}^{p}\right)^{2}+\left(u_{\tau}^{t}\right)^{2}} .
$$

Thus the skewed mean velocity profile is a direct consequence of the larger skin friction at the permeable wall than at the top wall.

The small wiggle near $z / H=-1$ in the profile of the DNS with cubes is caused by the change in the vertical extent of the averaging volume close to the solid wall.

\section{B. Rms profiles of velocity components and pressure}

The volume-averaged root-mean-square (rms) velocity $u_{i, \mathrm{rms}}$ is defined here according to

$$
\begin{aligned}
& u_{i, \mathrm{rms}} \equiv \sqrt{\left\langle\overline{\left\langle u_{i}^{\prime} u_{i}^{\prime}\right\rangle^{s}}\right.}
\end{aligned}
$$

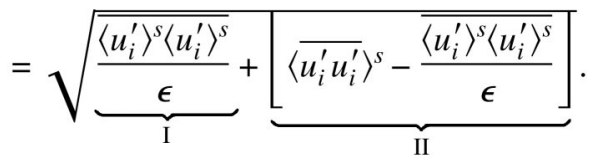

The decomposition of $u_{i, \mathrm{rms}}$ into the above two terms originates from the consideration that, when $\left\langle\left\langle u_{i}^{\prime}\right\rangle\right\rangle^{s} \approx\langle 1\rangle^{s}\left\langle u_{i}^{\prime}\right\rangle$ $=\left\langle u_{i}^{\prime}\right\rangle^{s}$ holds, term II is approximately equal to 

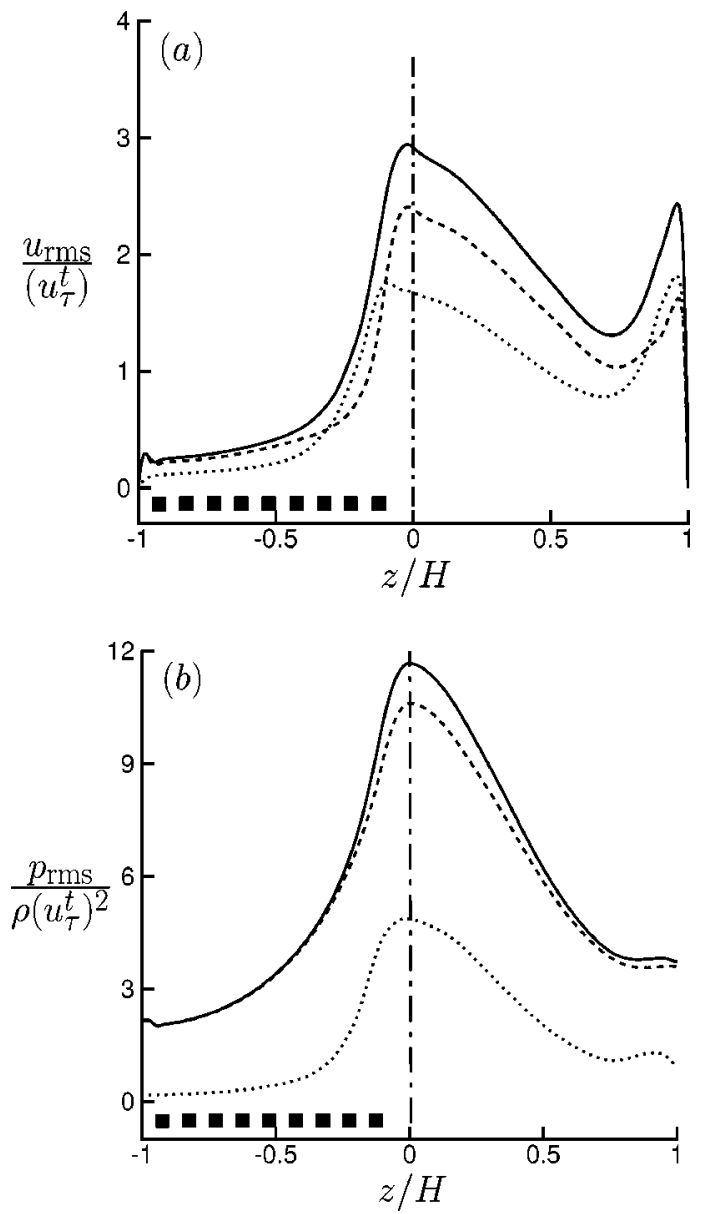

FIG. 11. Decomposition of the volume-averaged rms velocity and pressure according to Eq. (35) for the DNS with cubes. The black squares mark the position of the cubes. - , Total; --- contribution term I; ..., contribution term II. (a) Volume-averaged streamwise rms velocity; (b) volume-averaged rms pressure.

$$
\begin{aligned}
& \left\langle\overline{\left\langle u_{i}^{\prime} u_{i}^{\prime}\right\rangle^{s}}-\frac{\overline{\left\langle u_{i}^{\prime}\right\rangle^{s}\left\langle u_{i}^{\prime}\right\rangle^{s}}}{\epsilon}\right. \\
& =\overline{\left\langle\left[\left\langle u_{i}^{\prime}\right\rangle+\widetilde{u}_{i}^{\prime}\right]\left[\left\langle u_{i}^{\prime}\right\rangle+\widetilde{u}_{i}^{\prime}\right]\right\rangle^{s}}-\frac{\overline{\left\langle u_{i}^{\prime}\right\rangle^{s}\left\langle u_{i}^{\prime}\right\rangle^{s}}}{\epsilon} \\
& \approx \overline{\left\langle u_{i}^{\prime}\right\rangle\left\langle u_{i}^{\prime}\right\rangle}\langle 1\rangle^{s}+2 \overline{\left\langle u_{i}^{\prime}\right\rangle\left\langle\widetilde{u}_{i}^{\prime}\right\rangle^{s}}+\overline{\left\langle\overline{u_{i}^{\prime} \tilde{u}_{i}^{\prime}}\right\rangle^{s}}-\frac{\overline{\left\langle u_{i}^{\prime}\right\rangle^{s}\left\langle u_{i}^{\prime}\right\rangle^{s}}}{\epsilon} \\
& \approx \overline{\left\langle\widetilde{u}_{i}^{\prime} \widetilde{u}_{i}^{\prime}\right\rangle^{s} .}
\end{aligned}
$$

Thus, terms I and II represent basically the contributions of, respectively, the volume-averaged velocity $\left\langle u_{i}^{\prime}\right\rangle$ and the subfilter-scale velocity $\widetilde{u}_{i}^{\prime}$.

In the DNS with cubes, the contributions of both term I and term II in Eq. (35) can be calculated exactly. Figure 11 shows the result for $u_{\mathrm{rms}}$ and $p_{\mathrm{rms}}$. The contribution of the subfilter-scale flow field to $u_{\mathrm{rms}}$ is significant in the channel region and the top region of the grid of cubes, but appears to be small for $z / H \lesssim-0.5$. The rms pressure is dominated by the contribution of large-scale pressure fluctuations with a small contribution of the subfilter-scale pressure fluctuations throughout the flow domain.
In the DNS with continuum, just the volume-averaged flow field is resolved, and consequently only the contribution of term I can be calculated exactly. At this point we recall the discussion of the variable filter length in the DNS with continuum (see Secs. IV B and IV C). In the channel region it is assumed that the cellular-filter length is set by the mesh spacing, which is sufficiently small, and consequently term II is equal to zero in this region. In the homogeneous porous region, however, the cellular-filter length is equal to $l_{f}=d_{f}$ $+d_{p}$ and consequently in this region term II is not zero. As our main interest is in the behavior of the volume-averaged rms velocity in the channel region, we will not attempt here to model term II in the homogeneous porous region. Instead, in the DNS with continuum term II is simply put to zero.

The profiles of the volume-averaged rms velocities and pressure of the DNS with cubes, the DNS with continuum and the DNS with solid walls are depicted in Figs. 12(a)-12(d). The profiles of the DNS with cubes and the DNS with continuum compare very well. The differences between the profiles of these two simulations are small compared to the differences with the corresponding profiles of the DNS with solid walls. Notice that not only a good agreement exists between the DNS with cubes and the DNS with continuum in the channel region, but also inside the permeable wall, despite neglecting the contribution of term II in the DNS with continuum.

The rms profiles of the DNS with cubes and the DNS with continuum show an increase in all rms velocities and in the rms pressure near the permeable wall as compared to the profiles of the DNS with solid walls, at least when normalized by the friction velocity $u_{\tau}^{t}$ at the top wall. However, a more appropriate scaling of the rms profiles near the permeable wall is by means of the friction velocity $u_{\tau}^{p}$ at the permeable wall. Figure 13 shows the result for the rms profiles of the streamwise and the wall-normal velocity, respectively. The profiles are plotted as function of $z / \delta_{w}$, where $\delta_{w}$, given by Eq. (34), is considered as a characteristic lengthscale for eddies in the outer region of the boundary layer above the permeable wall. The peak in the streamwise rms velocity is smaller for the DNS with cubes and the DNS with continuum as compared to the DNS with solid walls. The large peak for the DNS with solid walls is associated with the presence of low- and high-speed streaks near a solid wall, which originate from the intense mean shear $(\partial\langle\bar{u}\rangle / \partial z)$ layer near the wall and the wall-blocking effect. The strong reduction in mean shear and the weakening of the wall-blocking effect prevent the formation of the streaks above a highly permeable wall, and this explains the decrease in the peak value of the streamwise rms velocity. The nonexistence of streaky structures near the permeable wall in the DNS with cubes and the DNS with continuum can be observed also in Fig. 8. The flow near the solid top wall is characterized by elongated streaky structures, whereas the flow near the permeable wall is dominated by relatively large-scale vortical structures. Similar vortical structures have been detected in experiments of flow over plant canopies, which originate from a Kelvin-Helmholtz type of instability of the inflexional mean velocity profile. ${ }^{24}$ The existence of these vortical structures is consistent with the dominant contribution from 

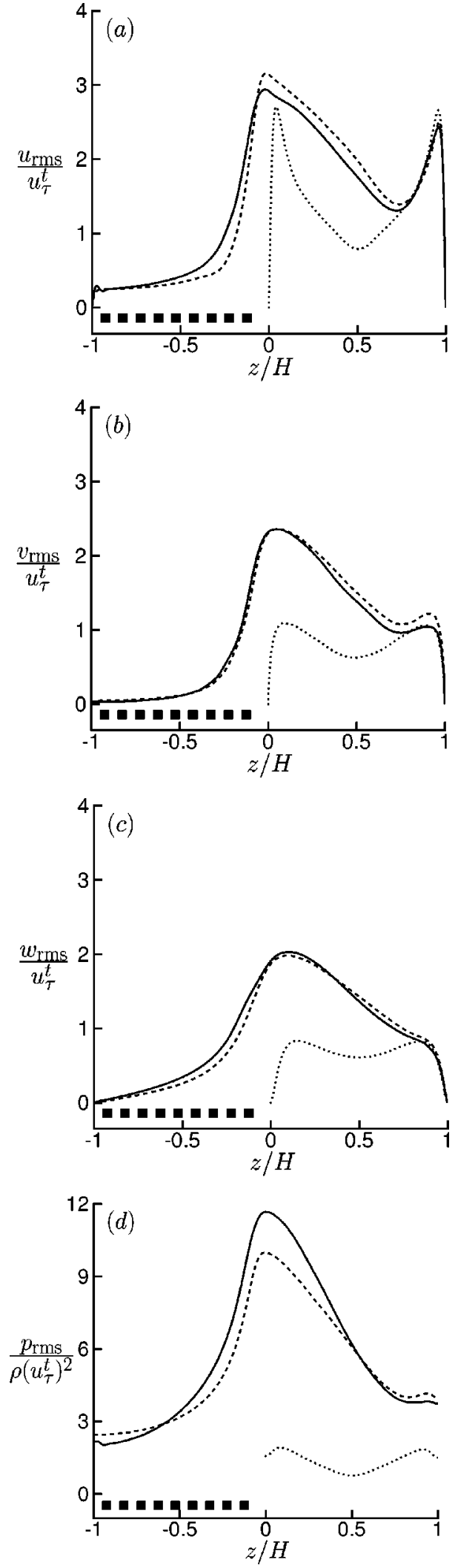

FIG. 12. Volume-averaged rms profiles of the velocity and pressure fluctuations in the DNS with cubes (-), the DNS with continuum (---), and the DNS with solid walls (...). The rms profiles are normalized by $u_{\tau}^{t}$ and $\rho\left(u_{\tau}^{t}\right)^{2}$, respectively, and plotted as function of $z / H$. The black squares mark the position of the cubes in the DNS with cubes. (a) Streamwise rms velocity; (b) spanwise rms velocity; (c) wall-normal rms velocity; (d) rms pressure.
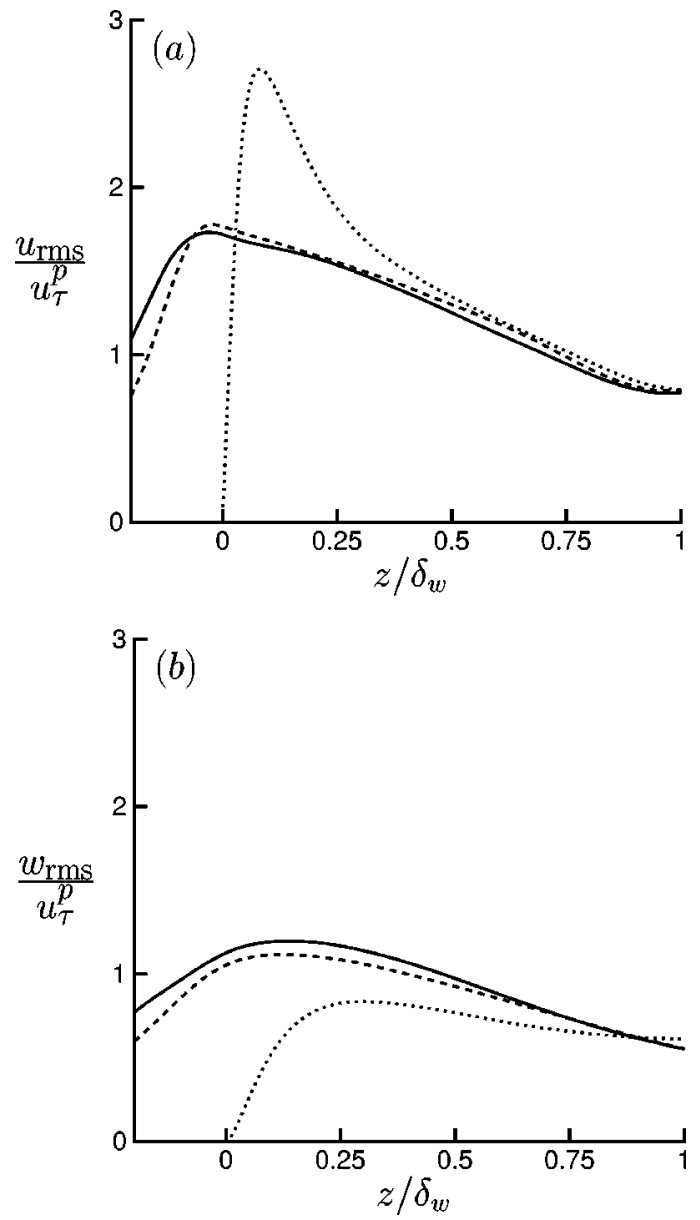

FIG. 13. Volume-averaged rms profiles of the streamwise and wall-normal velocity fluctuations in the DNS with cubes (-), the DNS with continuum (---), and the DNS with solid walls (...). The rms profiles are normalized by the friction velocity $u_{\tau}^{p}$ at the permeable wall and plotted as function of $z / \delta_{w}$. The black squares mark the position of the cubes in the DNS with cubes. (a) Streamwise rms velocity; (b) wall-normal rms velocity.

the volume-averaged flow field to the streamwise rms velocity around $z=0$ in Fig. 11(a) which is opposite to the behavior close to the solid top wall. Furthermore, as will be discussed in the following section, these vortical structures are also responsible for a strong increase in the Reynolds-shear stress at the permeable wall as compared to a solid wall. A more elaborate analysis of these vortical structures is beyond the scope of the present paper.

Opposite to the effect of wall permeability on the streamwise rms velocity, an increase is observed in the peak of the wall-normal rms velocity. This can be explained by the weakening of the wall-blocking effect, which no longer prohibits wall-normal transport of fluid across the wall interface. Notice that the profiles of the streamwise rms velocity overlap for $z / \delta_{w} \gtrsim 0.5$, thus exhibiting similarity, whereas in the same region the profiles of the wall-normal rms velocity of the DNS with cubes and DNS with continuum do not coincide with the profile of the DNS with solid walls.

\section{Shear-stress profiles}

The volume-averaged total shear stress $\left\langle\tau_{x z}\right\rangle^{s}$ is defined here as 


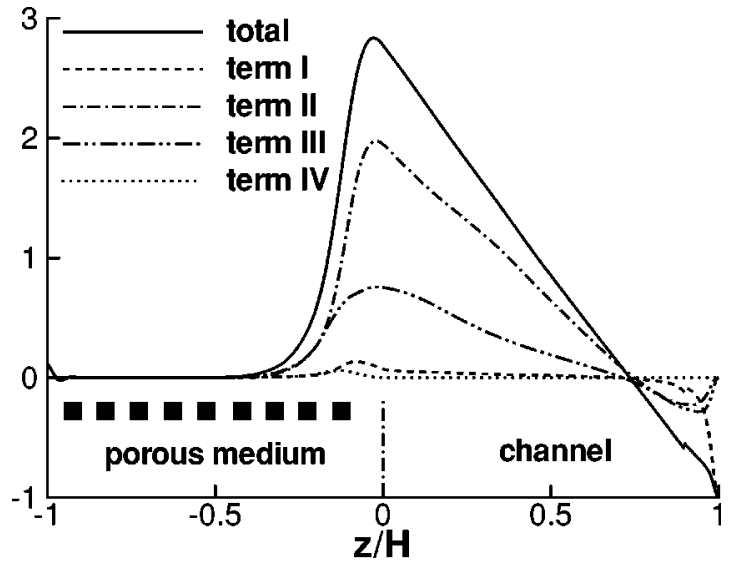

FIG. 14. Contribution of terms I-IV on the right-hand side of Eq. (36) to the volume-averaged total shear stress $\left\langle\tau_{x z}\right\rangle^{s}$ for the DNS with cubes. The stresses are normalized by $\left(u_{\tau}^{t}\right)^{2}$. The black squares mark the position of the cubes.

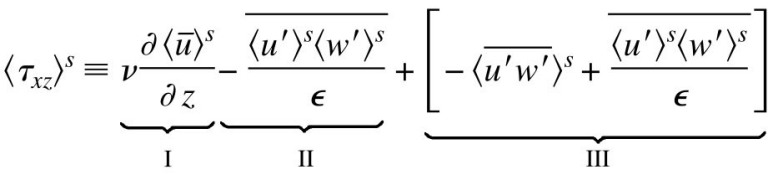

$$
\begin{aligned}
& \underbrace{-\langle\bar{u} \bar{w}\rangle^{s}}_{\text {IV }} .
\end{aligned}
$$

The terms on the right-hand side represent, respectively, the volume-averaged viscous shear stress (I), the contribution of large-scale motions (II) to the volume-averaged Reynoldsshear stress $\left\langle\overline{\left\langle u^{\prime} w^{\prime}\right.}\right\rangle^{s}$, the contribution of subfilter-scale motions (III) to the volume-averaged Reynolds-shear stress, and the volume-averaged mean shear stress (IV).

The terms in Eq. (36) have been calculated separately for the DNS with cubes. The result is shown in Fig. 14. The volume-averaged viscous shear stress (term I) peaks at the top wall due to the no-slip boundary condition, but it is negligible near the permeable wall. This substantiates the classification of the grid of cubes as a highly permeable wall. The kink in the profile of the volume-averaged viscous shear stress at $z / H=0.9$ is caused by the change in the vertical extent of the averaging volume close to the top wall, which leads to a small commutation error: $\langle\nu \partial \bar{u} / \partial z\rangle^{s} \neq \nu \partial\langle\bar{u}\rangle^{s} / \partial z$. The volume-averaged mean shear stress (term IV) is negligible throughout the flow domain. In most of the channel region the contribution of the large-scale motions (term II) is dominant over the contribution of the subfilter-scale motions (term III), but in the top region of the porous medium both contributions are equal. As mentioned before, at the end of Sec. VI B, the dominant contribution from the large-scale motions around $z=0$ is consistent with the presence of the relatively large-scale vortical structures observed in Fig. 8. These structures are responsible for an exchange of streamwise momentum between the channel region and the top layer of the permeable wall.

Figure 15 presents the profiles of the volume-averaged Reynolds-shear stress $\left\langle\overline{\left\langle u^{\prime} w^{\prime}\right.}\right\rangle^{s}$, viscous shear stress and total shear stress for the DNS with cubes, the DNS with con-
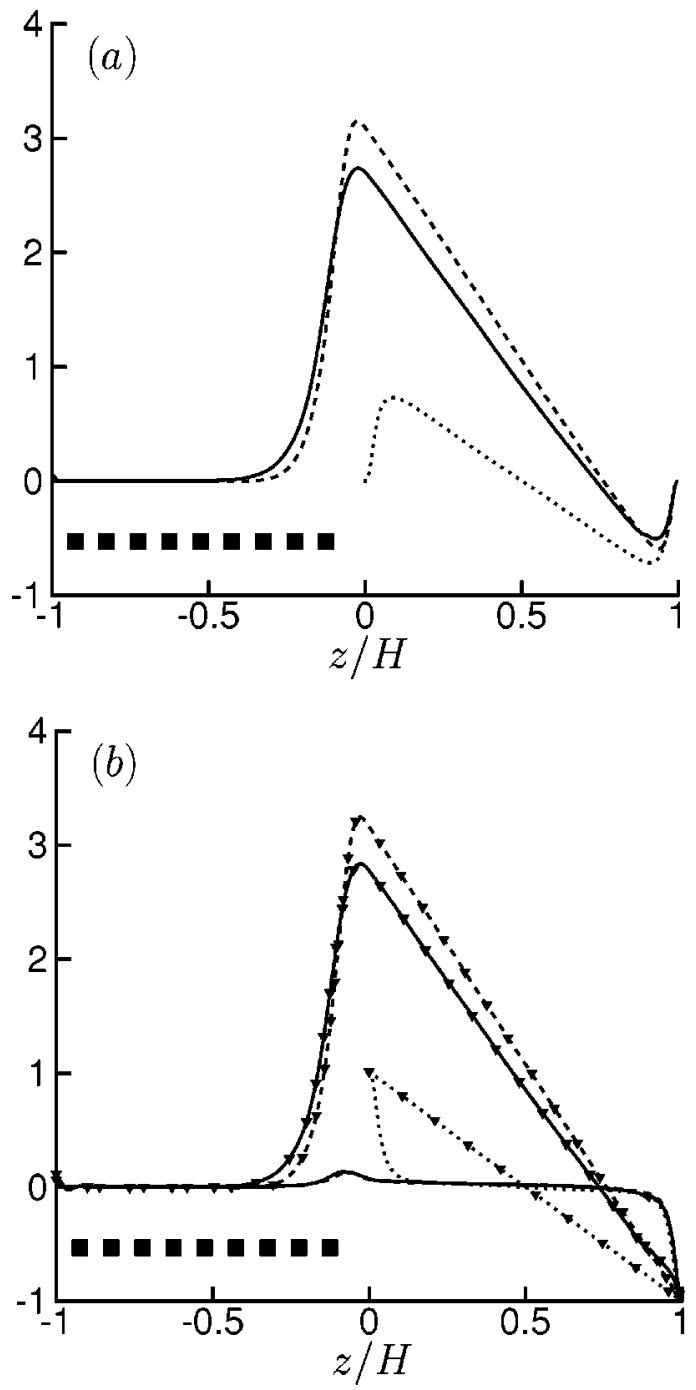

FIG. 15. Profiles of the volume-averaged Reynolds-shear, viscous shear, and total shear stress in the DNS with cubes (-), the DNS with continuum (---), and the DNS with solid walls (...). The stresses are normalized by $\left(u_{\tau}^{t}\right)^{2}$. The black squares mark the position of the cubes in the DNS with cubes. (a) Reynolds-shear stress; (b) viscous shear stress (lines without symbols) and total shear stress (lines with symbols).

tinuum and the DNS with solid walls. In the DNS with continuum, the contribution of the subfilter-scale motions (term III) to $\left\langle\overline{\left\langle u^{\prime} w^{\prime}\right.}\right\rangle^{s}$ and the volume-averaged mean shear stress (term IV) are neglected, based on a similar reasoning as given in the preceding section for the contribution of subfilter-scale motions to the volume-averaged rms velocity. The Reynolds-shear stress in the DNS with continuum is slightly overpredicted as compared to the DNS with cubes, although this discrepancy is small compared to the difference with the profile of the DNS with solid walls. With respect to the DNS with solid walls, we find a large increase in the Reynolds-shear stress for the DNS with cubes and the DNS with continuum, especially close to the permeable wall. This can be explained by a weakening of the wall-blocking effect, which no longer prohibits an exchange of streamwise momentum between the channel and the top layer of the porous medium by means of the previously observed vortical structures. Figure 15(b) shows that also a good agreement exists 
between the DNS with cubes and the DNS with continuum for the profiles of the volume-averaged viscous and total shear stress. As mentioned before, near the permeable wall the volume-averaged viscous shear stress is negligible. The large peak in the profile for the volume-averaged total stress is thus solely due to the strong increase in the volumeaveraged Reynolds-shear stress near the permeable wall.

\section{CONCLUSIONS AND DISCUSSION}

The main conclusion of the present study is that the continuum approach based the VANS equations is capable of an accurate simulation of the turbulent flow over and through a permeable wall, even quantitatively. In order to solve the VANS equations for the volume-averaged flow inside the permeable wall, closures are required for the subfilter-scale stress and the drag force. We have assumed a variable filter length for the DNS with continuum, with $l_{f}=d_{f}+d_{p}$ in the homogeneous porous region and $l_{f}=\Delta$ in the channel region. As argued by Breugem, ${ }^{5}$ subfilter-scale dispersion can usually be neglected in the homogeneous porous region. Because the mesh spacing $\Delta$ is sufficiently small, in the DNS with continuum subfilter-scale dispersion can be neglected in the channel region as well. In the literature many semiempirical relations exists for the drag force. In the present study a drag relation was adopted based on the Irmay $^{17}$ and the Burke-Plummer model, ${ }^{8}$ where the model coefficients were determined from simulations reported by Breugem et al. ${ }^{19} \mathrm{~A}$ variable-porosity model was used for the interface region to ensure that the VANS equations are continuous throughout the flow domain.

We have introduced the concept of a variable filter length for the DNS with continuum. In the processing of the DNS with cubes however, the filter length was kept constant to avoid commutation errors. Figure 14 has shown that in this case the volume-averaged mean shear stress $\langle\bar{u} \bar{w}\rangle^{s}$ can still be neglected, but that the contribution of the subfilterscale motions to the Reynolds-shear stress $\left.\overline{\left\langle u^{\prime} w^{\prime}\right.}\right\rangle^{s}$ may be important in the channel as well as in the interface region. In a previous study, ${ }^{5}$ we proposed a closure for the subfilterscale stress of the form $-\left\langle u_{i} u_{j}\right\rangle+\left\langle u_{i}\right\rangle\left\langle u_{j}\right\rangle=\left(K_{p}+K_{t}\right)\left(\partial\left\langle u_{i}\right\rangle / \partial x_{j}\right.$ $\left.+\partial\left\langle u_{j}\right\rangle / \partial x_{i}\right)$ with $K_{p}$ and $K_{t}$ the mechanical and turbulent viscosity, respectively. Although not evaluated in the present paper, this model seems to be promising.

The success of the continuum approach depends primarily on the accuracy of the closure for the drag force. In the present work we adopted the Irmay model for the range of low Reynolds numbers. This model is restricted to flow through a Cartesian grid of cubes and might not be suitable for other porous media. For example, for packed beds the Blake-Kozeny model ${ }^{8}$ is widely used. The coefficient in the Irmay model was determined from separate simulations through a fully periodic grid of cubes with a porosity of 0.875 . The value thus determined was close to the value of 12 proposed by Irmay for small porosities. This indicates that the Irmay model is valid for a large range of porosities, varying from almost zero to values close to unity. To model the drag force in the range of high Reynolds numbers, we adopted the Burke-Plummer model. This model performs well for many porous media, although the coefficient in this model might vary substantially dependent of the structure of the porous medium. MacDonald et al. ${ }^{8}$ found that a value of 1.8 is appropriate for many packed beds, while we found a value of about 0.4 for the Cartesian grid of cubes. The difference in these values is due to the fact that packed beds are disordered, whereas the Cartesian grid of cubes is ordered and aligned.

Other requirements for using the continuum approach are that the lengthscales of the volume-averaged and the subfilter-scale flow field are well separated, and that the permeability Reynolds number is relatively large. The former condition demands that the dimension of the pores and solid obstacles of the porous medium should be small compared to the channel height, because otherwise the flow in the channel region is more similar to flow around obstacles rather than flow over a permeable wall. Furthermore, the permeability Reynolds number should be relatively large to ensure that the effect of wall permeability on the turbulent flow in the channel dominates over the effect of surface roughness. The difference between wall permeability and wall roughness has been illustrated in Fig. 2. If the effect of surface roughness would be much more important, then it is not likely that the closure for the drag force in the interface region will give accurate results. This closure is namely based on the drag relation for uniform volume-averaged flow through a fully periodic porous medium, which will be quite different from the drag experienced by flow over the rough and impermeable wall in Fig. 2. Therefore, when surface roughness is important, one has to look for a more appropriate closure model for the drag force in the interface region. Instead of using the continuum approach, a better option in this case is probably a direct simulation, which captures the geometry of the surface roughness. A measure for the effect of surface roughness is the roughness Reynolds number. In the DNS with continuum this number was equal to 36.3 , see Table I, and according to Hinze ${ }^{11}$ this value falls the transitional roughness regime. The permeability Reynolds number was 13.5 and this value corresponds to the highly permeable regime..$^{5}$ This suggests that, in addition to the fact that the results of the DNS with cubes and the DNS with continuum agree well, in the present simulations the influence of wall permeability was strongly dominant over the effect of surface roughness. This suggestion is also supported by the relatively large rms velocities inside the permeable wall, and the important contribution of the volume-averaged Reynoldsshear stress to the total shear stress in the top layer of the permeable wall. We are aware that these arguments are only indirect evidence for the dominant effect of wall permeability over wall roughness. For a more conclusive claim concerning the importance of wall roughness with respect to wall permeability, we should actually simulate and compare the two cases shown in Fig. 2. This is however beyond the scope of this paper.

\section{ACKNOWLEDGMENTS}

This work was sponsored by the National Computing Facilities Foundation (NCF) for the use of supercomputer 
facilities, with financial support from the Netherlands Organization for Scientific Research (NWO). The authors would like to thank F. T. M. Nieuwstadt and R. E. Uittenbogaard for discussions on the manuscript.

${ }^{1}$ S. Hahn, J. Je, and H. Choi, "Turbulent channel flow with permeable walls," J. Fluid Mech. 450, 259 (2002).

${ }^{2}$ G. S. Beavers and D. D. Joseph, "Boundary conditions at a naturally permeable wall," J. Fluid Mech. 30, 197 (1967).

3 J. Bear, Dynamics of Fluids in Porous Media (Dover, New York, 1988).

${ }^{4}$ P. G. Saffman, "On the boundary condition at the surface of a porous medium," Stud. Appl. Math. L(2), 93 (1971).

${ }^{5}$ W. P. Breugem, "The influence of wall permeability on laminar and turbulent flows. Theory and simulations," Ph.D. thesis, Delft, 2004.

${ }^{6} \mathrm{~S}$. Whitaker, The Method of Volume Averaging (Kluwer, Dordrecht, 1999).

${ }^{7} \mathrm{M}$. Lesieur and O. Métais, "New trends in large-eddy simulations of turbulence," Annu. Rev. Fluid Mech. 28, 45 (1996).

${ }^{8}$ R. B. Bird, W. E. Stewart, and E. N. Lightfoot, Transport Phenomena (Wiley, New York, 2002).

${ }^{9}$ J. Happel and H. Brenner, Low Reynolds Number Hydrodynamics (Noordhoff International, Leyden, 1973).

${ }^{10} \mathrm{M}$. Quintard and S. Whitaker, "Transport in ordered and disordered porous media II: Generalized volume averaging," Transp. Porous Media 14, 179 (1994).

${ }^{11}$ J. O. Hinze, Turbulence (McGraw-Hill, New York, 1975).

${ }^{12}$ E. A. Fadlun, R. Verzicco, P. Orlandi, and J. Mohd-Yusof, "Combined immersed-boundary finite-difference methods for three-dimensional complex flow simulations," J. Comput. Phys. 161, 35 (2000).

${ }^{13}$ W. G. Gray, "A derivation of the equations for multi-phase transport,"
Chem. Eng. Sci. 30, 229 (1975).

${ }^{14}$ M. H. J. Pedras and M. J. S. De Lemos, "Macroscopic turbulence modeling for incompressible flow through undeformable porous media," Int. J. Heat Mass Transfer 44, 1081 (2001).

${ }^{15} \mathrm{~S}$. Whitaker, "Advances in theory of fluid motion in porous media," Ind. Eng. Chem. 61, 14 (1969).

${ }^{16}$ S. Whitaker, "The Forchheimer equation: a theoretical development," Transp. Porous Media 25, 27 (1996).

${ }^{17} \mathrm{~S}$. Irmay, "Modèles théoriques d'écoulement dans les corps poreux," Bulletin Rilem 29, 37 (1965).

${ }^{18}$ I. F. MacDonald, M. S. E-Sayed, K. Mow, and F. A. L. Dullien, "Flow through porous media: the Ergun equation revisited," Ind. Eng. Chem. 18, 199 (1979).

${ }^{19}$ W. P. Breugem, B. J. Boersma, and R. E. Uittenbogaard, "Direct numerical simulation of plane channel flow over a 3D Cartesian grid of cubes," Proceedings of the Second International Conference on Applications of Porous Media, edited by A. H. Reis and A. F. Miguel (Évora Geophysics Center, Évora, 2004), p. 27.

${ }^{20}$ J. H. Cushman, "Multiphase transport equations: I-General equation for macroscopic statistical, local, space-time homogeneity," Transp. Theory Stat. Phys. 12, 35 (1983).

${ }^{21}$ J. Kim, P. Moin, and R. Moser, "Turbulence statistics in fully developed channel flow at low Reynolds number," J. Fluid Mech. 177, 133 (1987).

${ }^{22} \mathrm{P}$. Wesseling, Principles of Computational Fluid Dynamics (SpringerVerlag, Berlin, 2001).

${ }^{23}$ J. Jimenez and P. Moin, "The minimal flow unit in near-wall turbulence," J. Fluid Mech. 225, 213 (1991).

${ }^{24}$ J. Finnigan, "Turbulence in plant canopies," Annu. Rev. Fluid Mech. 32, $519(2000)$ 